\title{
High Prevalence of Proarrhythmic Events in Patients With History of Atrial Fibrillation Undergoing a Rhythm Control Strategy: A Retrospective Study
}

\author{
Renato De Vecchis ${ }^{\mathrm{a}, \mathrm{f}}$, Marco Di Maio ${ }^{\mathrm{b}}$, Michel Noutsias ${ }^{\mathrm{c}}$, Angelos G. Rigopoulos ${ }^{\mathrm{c}}$, \\ Carmelina Ariano ${ }^{\mathrm{d}}$, Giuseppina Di Biase ${ }^{\mathrm{e}}$
}

\begin{abstract}
Background: A retrospective study was undertaken to evaluate the respective prevalence of proarrhythmic events depending on various therapeutic regimens within a population of patients with history of atrial fibrillation (AF) undergoing a rhythm control strategy.

Methods: Inclusion criterion was the presence of AF in the patient's clinical history, whose cardioversion had been followed by the adoption of rhythm control strategy. The primary endpoint was the determination of the respective prevalences of paradoxical arrhythmias in the various therapeutic groups. The secondary objective was all-cause mortality.

Results: A total of 182 cases of proarrhythmia out of 624 patients were detected during a median follow-up of 20 months (interquartile range: 18 - 24 months). The prevalences of proarrhythmic events were: IC antiarrhythmic drugs + beta-blockers, 111 cases out of a total of 251 patients $(44.22 \%)$; amiodarone, seven cases out of a total of 230 patients $(3 \%)$; sotalol, 61 cases out of a total of 140 patients $(43.57 \%)$; quinidine + digoxin, three cases out of a total of three patients $(100 \%)$. The paradoxical arrhythmias were: torsades de pointes, second- and third-degree sino-atrial block, slow atrial flutter with 1:1 atrioventricular (AV) conduction, second-degree Mobitz II AV block, and sustained monomorphic ventricular tachycardia. No fatal case of proarrhythmia was found.
\end{abstract}

Manuscript submitted March 5, 2019, accepted April 1, 2019

aPreventive Cardiology and Rehabilitation Unit, DSB 29 "S.Gennaro dei Poveri Hospital", Napoli, Italy

bDepartment of Cardiology, University of Campania "Luigi Vanvitelli", Naples, Italy

${ }^{\mathrm{c}}$ Mid-German Heart Center, Department of Internal Medicine III, Division of Cardiology, Angiology and Intensive Medical Care, University Hospital Halle, Martin-Luther-University Halle-Wittenberg, Halle (Saale), Germany

dDivision of Geriatrics, "Casa Sollievo della Sofferenza" Hospital, viale Cappuccini 2, San Giovanni Rotondo, Italy

eDivision of Geriatrics, Clinic "S. Maria del Pozzo", via Pomigliano 40, Somma Vesuviana, Italy

fCorresponding Author: Renato De Vecchis, Preventive Cardiology and Rehabilitation Unit, DSB 29 "S.Gennaro dei Poveri Hospital", via S.Gennaro dei Poveri 25, Napoli 80136, Italy. Email: devecchis.erre@virgilio.it

doi: https://doi.org/10.14740/jocmr3805
Conclusions: Secondary prevention of AF relapses by means of drugs suitable for accomplishing rhythm control strategy exposes the patients to incumbent risk of proarrhythmic events. Thus, the choice to avoid some varieties of antiarrhythmics with marked proarrhythmic potential (class IC drugs, sotalol, quinidine) appears to be warranted.

Keywords: Atrial fibrillation; Rhythm control strategy; Proarrhythmia

\section{Introduction}

Proarrhythmia is a term that collects in itself the broad spectrum of paradoxical arrhythmias connected by a causal relationship with the use of antiarrhythmic drugs [1].

Until recently, ventricular ectopic beats were the most common cause of the use of antiarrhythmics. Subsequently, it has been found that for the ventricular ectopic beats, even if frequent and repetitive (couples, triplets, burst or short episodes of non-sustained ventricular tachycardia), the use of antiarrhythmics may not be indicated in the presence of a structural heart disease with unfavorable remodeling, namely characterized by hypertrophy and/or dilatation of one or both ventricles. It has been observed that the most active antiarrhythmics in suppressing ventricular ectopies, namely Vaughan Williams class I and III drugs, are almost all burdened by a negative inotropic effect [2], which makes them contraindicated for ventricular ectopic beats in patients with reduced pump efficiency, except for amiodarone [3].

In contrast, the finding of life-threatening ventricular arrhythmias (e.g. history of sustained ventricular tachycardia (VT) episodes) or even the simple identification of a condition of decompensation with low $(\leq 35 \%)$ left ventricular ejection fraction (LVEF) persistent over time in spite of optimal decongestive therapy is an indication to the implantable cardioverter defibrillator (ICD) [4].

Thus, the paradoxical proarrhythmia from antiarrhythmics, related to prophylaxis or treatment of hyperkinetic ventricular arrhythmias, is now less frequent than in the past. In reality, today the most common indication for the use of antiarrhythmic drugs is the prophylaxis and/or treatment of atrial fibrillation (AF). In fact, this arrhythmia is very common, and 
Table 1. Vaughan Williams Classification, Comprising the Main Antiarrhythmic Medications

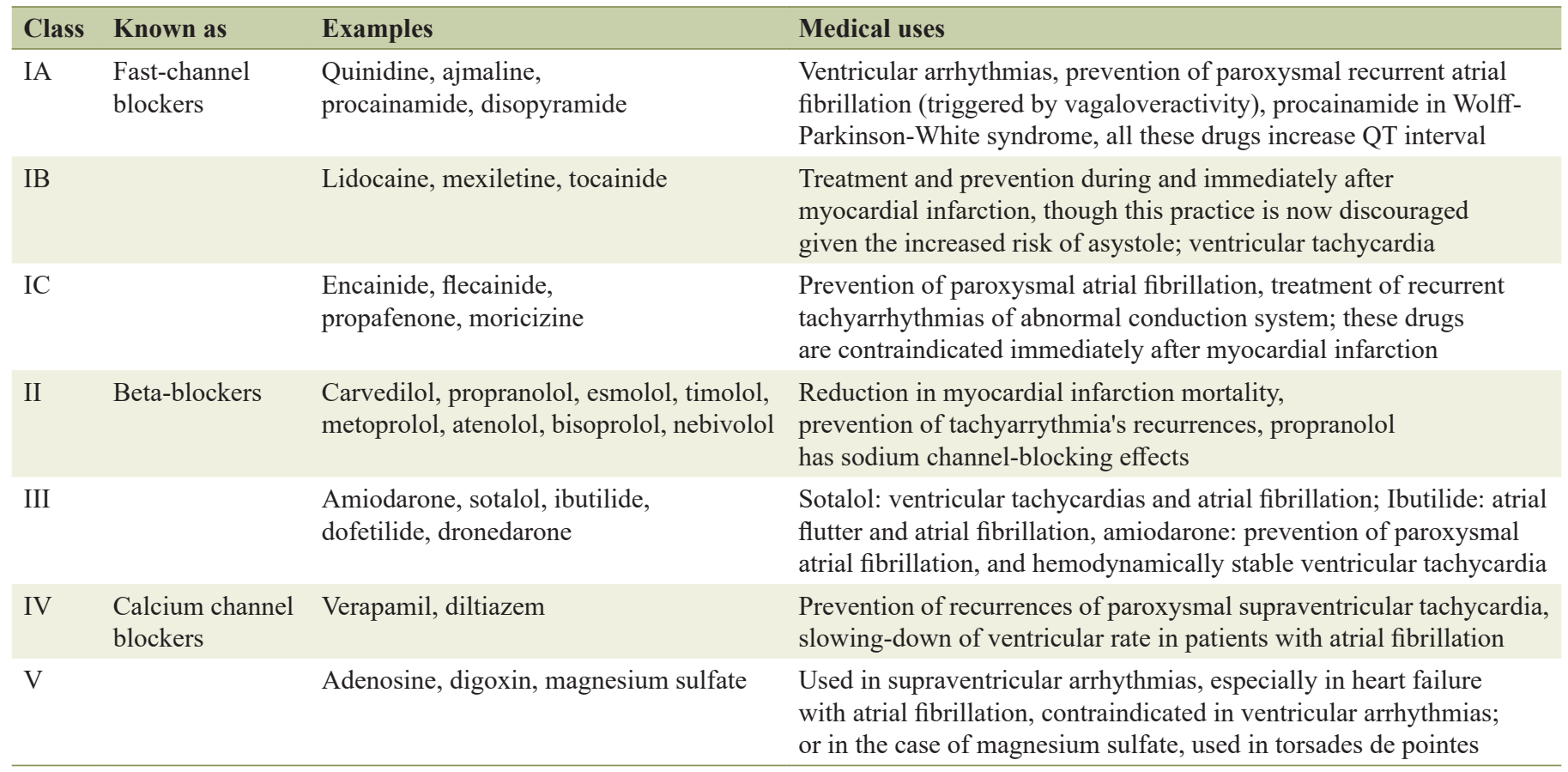

is still treated mainly with antiarrhythmic drugs rather than with trans-catheter ablation (ABL) today. Moreover, after retrieval of sinus rhythm, prevention of AF recurrences with chronic administration of antiarrhythmics would appear as a useful choice, according to some publications [5-6].

Thus the therapies for newly diagnosed AF, such as external electrical cardioversion, intravenous (IV) or oral pharmacologic cardioversion, $\mathrm{ABL}$, which have restored sinus rhythm, are frequently followed by a secondary prophylaxis of AF recurrences, carried out with class I or III drugs (Vaughan Williams classification) [5-6]. In this regard, a thorough and detailed representation of the current classification of the antiarrhythmics is contained in the Table 1 . We recommend to refer to this table for acquiring a summary information about the properties of these electrophysiologic agents.

Based on retrospective evaluation of inpatient and outpatient clinical records collected from two hospitals, our study has addressed the problem of identification of proarrhythmic events that are related to use of antiarrhythmic drugs prescribed for prevention of AF relapses, within the scope of the approach known as "rhythm control strategy".

The primary purpose was to detect the respective prevalence of different types of paradoxical arrhythmia depending on the antiarrhythmic drug treatment. A secondary objective was to verify whether proarrhythmia could cause a higher allcause mortality.

\section{Materials and Methods}

In our retrospective study the cases of proarrhythmia were identified taking several criteria into account: 1) There had to be a high probability regarding the existence of a causal relationship between the assumption of the antiarrhythmic drug and the onset of the paradoxical arrhythmia; 2) A cardiopathy had to be present; 3) A definite diagnosis of the arrhythmia must have been made; and 4) The possible interruption of the antiarrhythmic treatment should be followed by the disappearance or attenuation of the paradoxical arrhythmia.

Our retrospective study used a series of patients all characterized by the availability of the relevant electrocardiographic documentation, and by anamnestic data suitable for demonstrating the pathogenetic dependence of the arrhythmia on the prescribed antiarrhythmic drugs. The study population included patients with a history of paroxysmal, persistent or long-lasting persistent AF. Inclusion criterion was as follows: patients who achieved sinus rhythm with external transthoracic electrical shock or pharmacological cardioversion or trans-ABL had to comply with a pharmacological prevention strategy of AF recurrences (so-called rhythm control strategy).

In this setting of patients with a history of previous paroxysmal, persistent or long-lasting persistent AF, the respective prevalences of the various iatrogenic arrhythmias were calculated for each of the antiarrhythmic regimens aimed at preventing $\mathrm{AF}$ recurrences.

The pertinent cases were collected through the retrospective evaluation of inpatient and outpatient clinical records and the attached electrocardiograms coming from the Cardiology Division of the "Casa Sollievo della Sofferenza" Hospital (San Giovanni Rotondo, Italy), as well as from the Division of Geriatrics of the Clinic "S. Maria del Pozzo" (Somma Vesuviana, Italy). The authors of the retrospective research received the necessary information and documentation upon agreement with the respective Hospital Health Directorates. 
The agreement provided for access by the authors to the consultation of medical sheets, available in electronic or paper format, and the faculty to evaluate the clinical diaries of the outpatients, as well as the related electrocardiograms attached. Priority was given to the protection of privacy and preservation of anonymity. Indeed, the collection of aggregated data was conducted in such a way that it was impossible to trace the identity of the involved patients.

The patients were also asked to give their informed consent to the investigation conducted on their clinical documentation, after being traced and interviewed by telephone interview. Retrospective research involved proarrhythmic events concerning the 2013 - 2017 period. Whenever a certain or suspected case of proarrhythmia was detected, a researcher cared about recording the case, and about collecting a copy of the relevant electrocardiogram. Importantly, physicians who prescribed the antiarrhythmic drugs possibly involved in an episode of proarrhythmia did not coincide with the research authors. So the authors were completely unrelated to the choices and therapeutic criteria adopted by the treating physicians, and their activity consisted of a mere work of transcription, collection and statistical elaboration of clinical data.

\section{Statistical analysis}

Continuous data are reported as means $( \pm$ SD) or medians (interquartile range (IQR)). Kolmogorov-Smirnov test was used to test for normality. Comparisons between groups were performed using the Student's $t$-test or Mann-Whitney U test where appropriate. Categorical data were reported as proportions or percentages and their intergroup comparisons were performed using the Chi-square test or the Fisher's exact test where appropriate. Likewise, when necessary, Kruskal-Wallis as well as one-way analysis of variance (ANOVA) tests were carried out.

Analyses were performed according to the intention-totreat principle. The primary endpoint, namely the respective frequency of the paradoxical arrhythmias for each of the pharmacologic regimens was represented by means of percentages. Statistical tests were two tailed, and $\mathrm{P}$ value $\leq 0.05$ was considered statistically significant. Analyses were conducted using Excel 2016 Version 16.0 (Seattle, WA, USA) as well as MedCalc Version 18.6 (Acacialaan 22, 8400 Ostend, Belgium).

Institutional Review Board Approval was not required, due to retrospective nature of the work. This study was conducted in compliance with the ethical standards of the responsible institution on human subjects as well as with the Helsinki Declaration.

\section{Results}

A total of 182 cases of proarrhythmia were identified (Table 2) within a total population of 624 patients (29.1\%) with AF history, all subjected to rhythm control strategy for secondary prophylaxis of AF relapses. In $20 \%$ of the cases equal to 125 patients, the rhythm control strategy was adopted to
Table 2. The Absolute Number of Cases for Each Paradoxical Arrhythmia Variety

Torsades de pointes: 56 cases

Slow atrial flutter with 1:1 AV conduction: 24 cases

Intermittent high grade AV block in patients with

asymptomatic chronic bifascicular block: one case

Left bundle branch block: 23 cases

Right bundle branch block: 13 cases

Second-degree sino-atrial block type 1: five cases

Second-degree sino-atrial block type 2: 45 cases

Third-degree sino-atrial block: 31 cases

Type 2 (Mobitz II) second-degree AV block: 14 cases

Sustained monomorphic ventricular tachycardia: six cases

On the whole, there are 182 cases of paradoxical arrhythmia (proarrhythmic events) plus 36 cases of iatrogenic intraventricular conduction delay, namely 23 cases of left bundle branch block and 13 cases of right bundle branch block caused by antiarrhythmic drugs, especially flecainide and quinidine.

supplement the previous AF ABL. The median follow-up of this retrospective research, relating to the time frame 2014 2017, was 20 months (IQR: 18 - 24 months). Pharmacological prophylaxis for rhythm control was accomplished with the use of various drugs and dose schedules.

A first approach entailed the use of Vaughan Williams IC class drugs (251 patients, 40.2\%), namely propafenone, 450 - $600 \mathrm{mg}$ per day or flecainide, 100 - $200 \mathrm{mg}$ per day, with daily dose divided into two administrations. Usually the IC class drugs were supplemented by the addition of beta-blockers (BBs) at low doses, namely bisoprolol 2.5 - $5 \mathrm{mg}$ per day or carvedilol 12.5 - $25 \mathrm{mg}$ per day or metoprolol $25-50 \mathrm{mg}$ per day, with daily dose divided into two administrations. A second scheme, also aimed at the preservation of the rhythm, provided for amiodarone (Vaughan Williams class III), $200 \mathrm{mg}$ per day (230 patients, 36.8\%). A further alternative was the administration of sotalol (class III), $80 \mathrm{mg}$ twice daily (140 patients, $22.4 \%$ ). Three patients were treated with hydroquinidine hydrochloride (Vaughan Williams class IA) $150 \mathrm{mg}$ twice daily plus digoxin $0.125 \mathrm{mg}$ once daily.

In the subset of patients undergoing ABL, for whom a prophylaxis with antiarrhythmics had been decided after the blanking period (125 patients), the rhythm control strategy was always constituted by the use of a class IC drug (flecainide or propafenone at the above mentioned doses) corroborated by the additional administration of small doses of BB (bisoprolol or metoprolol at the above mentioned doses). Thus, the ablated patients (no. 125) accounted for about half of the entire patient subset assigned to IC + BB therapy (251 patients on the whole).

The distribution of 182 proarrhythmic events through the four subsets $(\mathrm{IC}+\mathrm{BB}$, amiodarone alone, sotalol alone, quinidine plus digoxin) is shown in Figure 1.

Based on the histograms in Figures 2-4, the IC antiarrhythmics were characterized by a very high risk of hypokinetic arrhythmias, most notably constituted by the second-degree type 


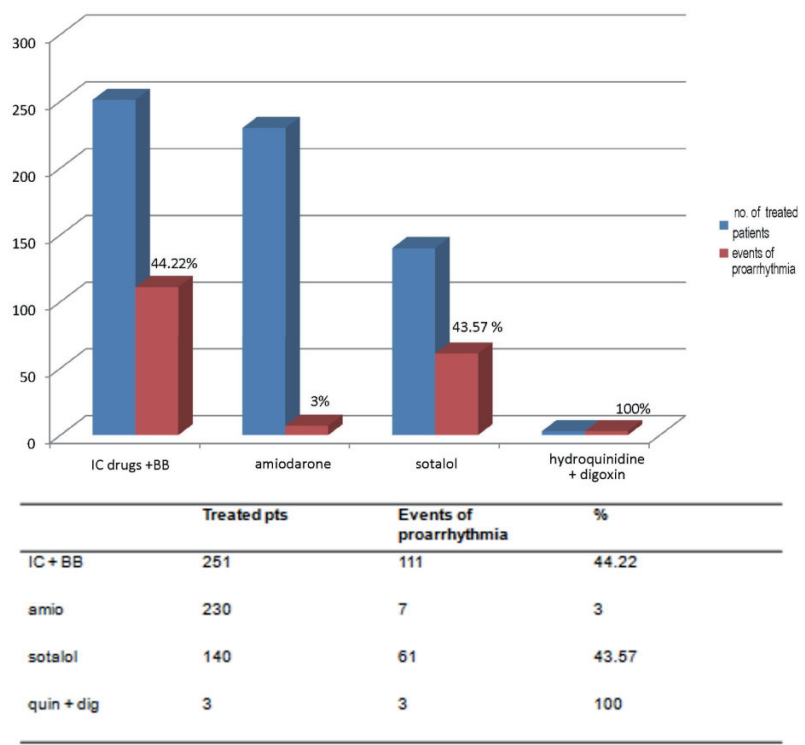

Figure 1. The respective percentages of events of proarrhythmia, depending on the type of drug antiarrhythmic treatment for prophylaxis of AF relapses, are depicted (purple bars). AF: atrial fibrillation; IC: antiarrhythmics belonging to class IC of Vaughan Williams classification; BB: beta-blockers; amio: amiodarone; quin: hydroquinidine; dig: digoxin.

2 sino-atrial block and the third-degree sino-atrial block (50 cases of sino-atrial block of various degree out of a total of 251 treated patients, equal to $19.92 \%$, Fig. 2, left panel). For IC antiarrhythmics there was also a risk of second-degree Mobitz II atrioventricular (AV) block (5.57\% equal to 14 cases out of a total of 251 treated patients, Fig. 3, right panel). Furthermore, the risk of torsades de pointes $(7.96 \%$, Fig. 3 , left panel) has been documented for IC therapy.

Regarding the three registered cases $(1.19 \%)$ of sustained monomorphic VT (Fig. 4), the attribution of a causal role to IC drugs required in each case an in-depth discussion among coauthors. Indeed, a causal role of antiarrhythmics was questioned by some, because the patients were all suffering from severe cardiac pathology which per se might be involved in the genesis of these VT episodes. In addition, 22 patients out of a total of $251(8.76 \%)$ showed a typical arrhythmia of IC patients, namely slow atrial flutter (AFL) with 1:1 AV conduction.

As a general therapeutic measure, when any proarrhythmic event was identified, the discontinuance of therapy with IC drugs (flecainide or propafenone) was carried out, except for the cases of AFL with 1:1 AV conduction, see beyond. In addition, in many cases of proarrhythmia, the hospitalization for close clinical and instrumental surveillance was required.

For patients of the IC group with abnormal drug-related sinus automatism (50 out of a total of 251 patients), implantation of a transient pacemaker was needed in 10 cases of marked bradyarrhythmia (mean frequency $\leq 35$ beats per min). Among them, following thorough electrophysiologic study, five patients underwent definitive permanent pacemaker (PM) (atrial demand pacemaker (AAI) or rate-responsive dual chamber pacemaker (DDD)) implantation.

Among the 14 cases of Mobitz II second-degree AV block, there was a rapid regression of the arrhythmic disorder in 10 cases, requiring only a period of hospital stay with close clinical and instrumental observation. Conversely, the finding of infra-Hisian conduction delay persistent over time in four patients, coupled with chronotropic incompetence, prompted physicians to implantation of a definitive pace-maker (DDD-R in all cases).

In patients treated with IC drugs, the symptomatology of
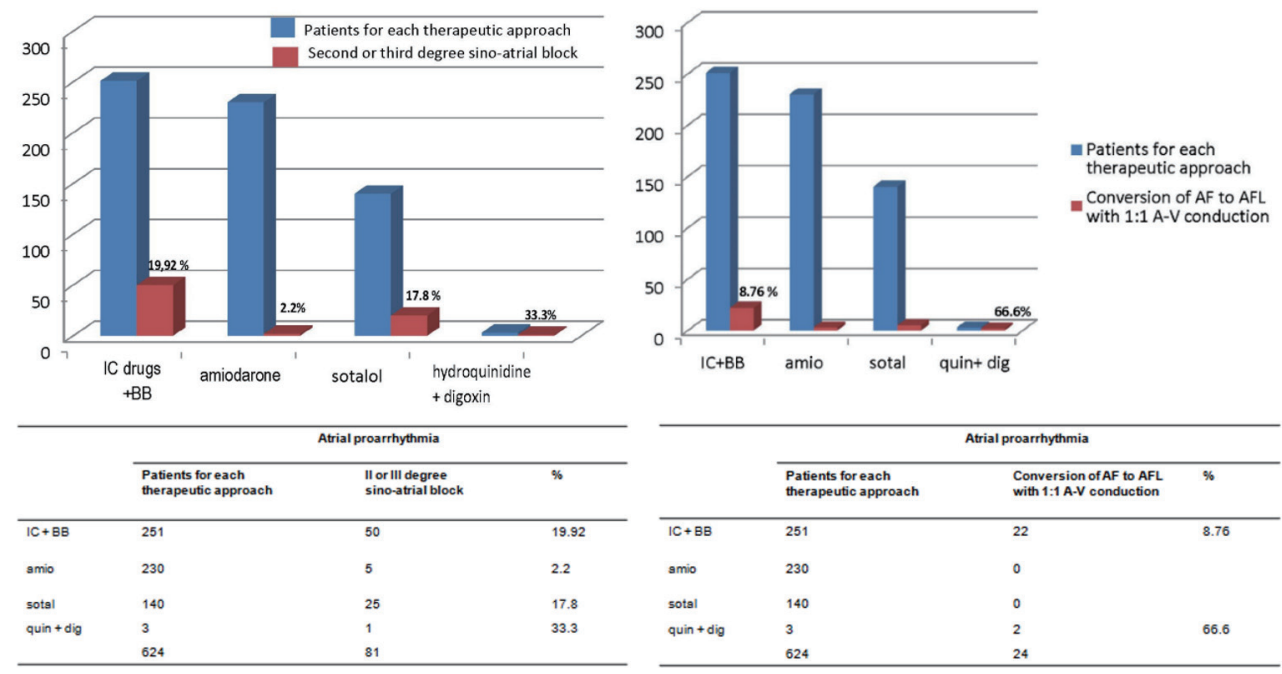

Figure 2. The respective percentages of events of atrial proarrhythmia, depending on the type of drug antiarrhythmic treatment for prophylaxis of AF relapses, are reported (purple bars). The histograms refer to two atrial proarrhythmia events with important clinical correlates, namely the second- or third-degree sino-atrial block (left panel) and the atrial flutter with AV 1:1 conduction (right panel). AF: atrial fibrillation; AFL: atrial flutter; AV: atrioventricular; IC: antiarrhythmics belonging to class IC of Vaughan Williams classification; BB: beta-blockers; amio: amiodarone; quin: hydroquinidine; dig: digoxin. 


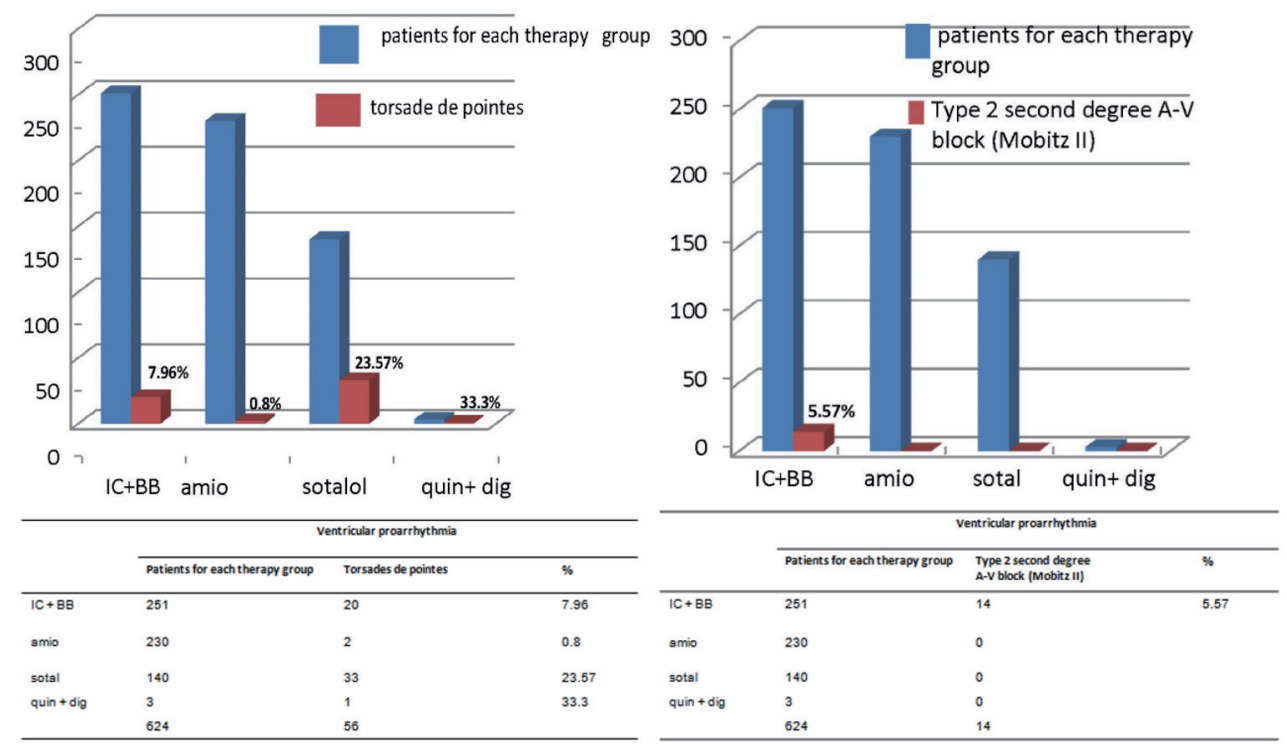

Figure 3. The respective percentages of torsades de pointes and type 2 second-degree AV block, depending on the type of drug antiarrhythmic treatment for prophylaxis of AF relapses, are represented (purple bars). All of the cases of type 2 second-degree AV block (n. 14) are attributable to IC + BB therapy. As regards the torsades de pointes the risk appears to be more pronounced when using hydroquinidine plus digoxin (33.3\%) or sotalol (23.57\%). AV: atrioventricular; IC: antiarrhythmics belonging to class IC of Vaughan Williams classification; BB: beta-blockers; amio: amiodarone; quin: hydroquinidine; dig: digoxin.

polymorphic VT with torsades de pointes consisted of dizziness and weakness (12 cases) whereas syncope was detected in eight cases. Importantly, the bursts of torsades de pointes have never resulted in ventricular fibrillation. Likewise, no neuro-

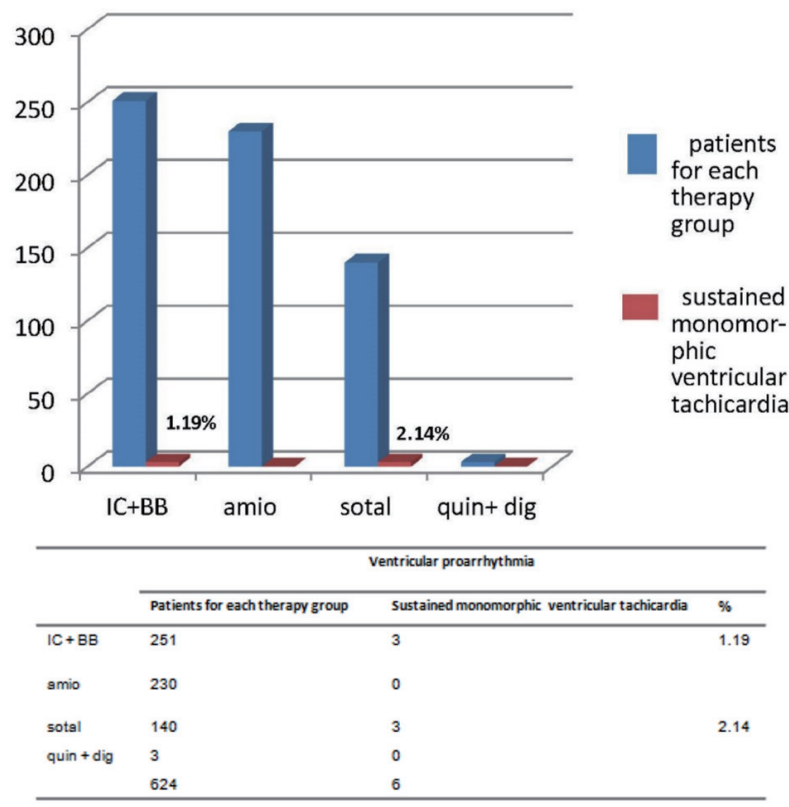

Figure 4. The respective percentages of sustained monomorphic ventricular tachicardia, depending on the type of drug antiarrhythmic treatment for prophylaxis of AF recurrences, are highlighted (purple bars). This very dangerous arrhythmia has been found in IC + BB group (1.19\% of the treated patients) and sotalol group (2.14\%). For further considerations, please see the text. logical sequelae have been noticeable on clinical examination in all cases. Torsades de pointes were treated in 15 patients with IV lidocaine at low doses $(4 \mathrm{mg} / \mathrm{min}$ for $2 \mathrm{~h} 30 \mathrm{~min}$, then an additional $600 \mathrm{mg}$ in $5 \mathrm{~h}-2 \mathrm{mg}$ per min). For the management of torsades de pointes in five out of a total of 20 IC patients with this arrhythmia, IV administration of magnesium sulfate $(2$ - $4 \mathrm{mg} / \mathrm{min}$ ) was preferred.

For the three detected cases of sustained VT attributed to therapy with IC + BB drugs, cessation of the arrhythmia was achieved by IV lidocaine infusion ( $4 \mathrm{mg} / \mathrm{min}$ ) with an average total dose of $600 \mathrm{mg} /$ patient. The cases of slow AFL with 1:1 AV conduction-a type of paradoxical arrhythmia found exclusively in patients treated with IC drugs, usually occurred during $\mathrm{AF}$ relapses, arising from transient transformation of $\mathrm{AF}$ into AFL, with $\mathrm{F}$ waves transmitted to the ventricles without AV block interposition. In any case, IV therapy with verapamil $2.5 \mathrm{mg}$ given as a single or repeated bolus was adopted. The subsequent clinical course was always favorable with restoration of sinus rhythm in all cases within $12 \mathrm{~h}$. The IC drug was usually maintained with the addition of oral verapamil $(40 \mathrm{mg}$ twice daily) in place of BB. This empirical approach was based on the concept that it was appropriate to replace $\mathrm{BB}$ with a different negative dromotropic agent such as verapamil, considering the inability of the former in these cases to prevent the AFL with 1:1 AV conduction.

Among the proposed pharmacological algorithms for AF prophylaxis, amiodarone alone at dose of $200 \mathrm{mg}$ per day appeared to have a favorable risk/benefit ratio, being characterized by only seven proarrhythmic events $(3 \%)$ out of a total of 230 treated patients.

Sotalol was characterized by a high percentage of proarrhythmic events (61 out of a total of 140 treated patients: $43.57 \%$ ). The qualitative composition was as follows: 33 cases 
(23.57\%) of torsades de pointes, $25(17.8 \%)$ cases of second or third-degree sino-atrial block, and three cases of sustained monomorphic VT. Torsades de pointes entailed dizziness, lipothymia or syncope.

Third-degree sino-atrial block was accompanied by a sense of asthenia, exertional dyspnea due to chronotropic incompetence, faint and dizziness. Sustained monomorphic VT resulted in syncope in all cases and its interruption was induced by the timely administration of lidocaine as a single bolus of $1 \mathrm{mg} / \mathrm{kg}$ in one case or by means of IV infusion (4 $\mathrm{mg} / \mathrm{min}$ ) in two cases. No sotalol-related proarrhythmia event required external transthoracic electrical shock. Similarly no pacemaker or ICD implant was needed due to sotalol administration. The quinidine-digoxin association induced more than one proarrhythmic event: in a patient both torsades de pointes and the third-degree sino-atrial block, arising in succession as documented by the continuous electrocardiogram (ECG) recording in patient with ICD; in two patients, AFL with 1:1 AV conduction.

These arrhythmias did not require any specific measure other than discontinuation of quinidine-digoxin combination therapy and its replacement with amiodarone $200 \mathrm{mg}$ per day.

The cases of death identified by our retrospective study were 15 out of a total of 624 patients adhering to a rhythm control strategy $(2.4 \%)$. Proarrhythmic events have never been involved in these deaths. In fact, the causes of death were: irreversible progression of chronic heart failure (seven cases), myocardial infarction (two cases), pulmonary embolism consequent to deep vein thrombosis (one case), ischemic stroke from lacunar cerebral infarction (one case), bacterial endocarditis during sepsis (one case), chordae tendineae rupture complicating mitral prolapse (one case), bacterial peritonitis complicating ascites secondary to heart failure (one case), and subarachnoid hemorrhage (one case).

\section{Discussion}

A salient feature was the fact that the study was limited to AF patients undergoing the therapeutic approach known as rhythm control strategy. Importantly, the proarrhythmic events have exhibited a relatively high prevalence in our study $(29.1 \%)$. This might mark that antiarrhythmic therapy has been poorly conducted, or that it has not been suitable for the patient's needs.

Indeed, in our experience the rhythm control strategy, being aimed at preventing AF recurrences, has exhibited a different proarrhythmic potential depending on the type of prescribed antiarrhythmic drug. In particular, IC drugs of the Vaughan Williams classification exhibited a high risk of proarrhythmic events since they induced 111 arrhythmic episodes out of a total of 251 treated patients (44.22\%) (Fig. 1).

In the histograms represented in Figures 2-4, where the distribution of arrhythmias is graphically described according to the therapeutic regimen, it is noticeable that IC drugs are encumbered by a high risk of hypokinetic arrhythmias such as sino-atrial (Fig. 2, left panel) and AV (Fig. 3, right panel) blocks, in addition to being associated with non-negligible risk of torsades de pointes, even if to a lesser extent compared to sotalol.

Perhaps the combined therapy (IC drugs plus small doses of $\mathrm{BBs}$ ) adopted for preventing a possible AFL with 1:1 AV conduction might have unveiled a sick sinus syndrome in some patients.

It is very intriguing to note that about half ( 125 cases) of the group treated with IC antiarrhythmic drugs (no. 251) was composed of patients undergoing ABL. In this regard, it should be mentioned that for a long time, ABL had been presented as an approach not only efficacious for AF conversion to sinus rhythm, but also suitable for achieving a durable rhythm normalization without the need to resort to the use of antiarrhythmic drugs to maintain the result achieved [7]. These assertions were then denied by the finding that long-term administration of antiarrhythmic drugs-for the twelve months following ABL or even for longer periods of time [5,8]-increased the likelihood of AF prevention in the long term. In fact, the incidence of AF recurrences was shown to be significantly lower in ablated patients assigned to antiarrhythmic drug treatment than in those who did not receive antiarrhythmics [6]. Furthermore in patients with recent ABL, a relatively low efficacy of amiodarone for the prevention of AF recurrences was highlighted [9], with consequent indication to preferably use IC drugs or sotalol for this patient subgroup.

Really, the practice of preferably using IC or IA antiarrhythmic drugs or sotalol as a pharmacological support for $\operatorname{ABL}[6,10,11]$ is now a customary and established habit. In fact, as already mentioned, in our patient population, the whole subset of ablated patients $(n=125)$ received IC antiarrhythmic drugs.

Indeed, according to current guidelines [12], ABL should be granted for AF patients with marked symptoms in whom at least one antiarrhythmic drug has been unsuccessfully tested. So it is reasonable to argue that among the 251 patients receiving IC drugs, the 125 ablated patients might be the patients with highest risk of $\mathrm{AF}$ relapses.

The retrospective nature of the research has made it possible the confounding by indication, namely patients more prone to $\mathrm{AF}$ recurrences and with more severe clinical picture were more likely to be assigned to treatment with IC drugs or sotalol. Therefore, the results have to be analyzed with caution.

It is interesting to note that paradoxical arrhythmias have not been so severe as to induce an increase in mortality in involved patients over a median follow-up of 20 months (IQR: 18 - 24 months).

Deaths were in no case attributable to proarrhythmic events. However, proarrhythmia led to the identification of patients with latent disorders of the genesis and/or conduction of electrical impulse. They were addressed to definitive electrostimulation: five for sick sinus syndrome and four for Mobitz II second-degree AV block. So proarrhythmia has led physicians to perform electrophysiological studies that have caused the implantation of pacemakers. In a certain sense, in these cases the proarrhythmic events have functioned as a markers of hidden disorders, thereby allowing the identification of more vulnerable patients. It is possible that patients with latent dysfunction of the specific conduction tissue are more prone to proarrhythmic events, especially hypokinetic atrial arrhythmias. 
On the whole, the major results of our study could be interpreted as follows: 1) Antiarrhythmic drugs belonging to classes IA (quinidine) and IC (propafenone, flecainide) as well as the sotalol included in the class III of Vaughan Williams classification are characterized by considerably frequent events of proarrhythmia, and this would require reconsidering their current indication for long-term prophylaxis of AF relapses; and 2) Class IC antiarrhythmics are likely to be largely used for purposes of obstinate preservation of sinus rhythm in patients treated with ABL, albeit severe remodeling of atrial chambers in many cases would rather suggest to abandon the therapeutic target of rhythm control in favor of a strategy of judicious anticoagulation coupled with concomitant administration of negative dromotropic agents (BB or verapamil) to accomplish the socalled rate control strategy. This renunciation of cardioversion would be counterbalanced by the advantage of being exempted by therapies likely to elicit paradoxical arrhythmias, such as therapies with IC drugs or sotalol. Moreover, rate control strategy has been proven to yield a similar survival and a superior quality of life compared to rhythm control strategy [13].

However this interpretation, elaborated within the frame of a retrospective study, should be validated by a prospective randomized controlled trial suitable for comparing the respective odds of death of two groups of patients with history of AF, the former being left free from IC antiarrhythmics and sotalol and the latter undergoing pharmacological prophylaxis with these antiarrhythmics.

Additionally, amiodarone given at dose of $200 \mathrm{mg}$ per day has been shown to be safe and devoid of risk of proarrhythmia, thereby justifying its use for AF prophylaxis. However, its efficacy is deemed poor in many cases of AF, especially for patients undergoing $\mathrm{ABL}$, who are more prone to $\mathrm{AF}$ recurrences [9].

\section{Study limitations}

The retrospective collection of a data set constitutes the most frequently used method for gathering medical health information, but at the same time it implies an important limitation, because it entails relatively frequent biases. This also applies to our study and should advise caution in the interpretation of the results. Further limitations of our study are the rather exiguous sample size and the relatively short duration of the follow-up.

\section{Conclusions}

Secondary prevention of AF relapses exposes the patients to incumbent risk of proarrhythmic events. Thus, the choice to avoid some varieties of antiarrhythmics with marked proarrhythmic potential (IC drugs, sotalol, quinidine) appears to be warranted.

\section{Acknowledgments}

None.

\section{Financial Disclosure}

The study has not benefitted from any source of funding.

\section{Conflict of Interest}

Michel Noutsias has received grants from the Deutsche Forschungsgemeinschaft (DFG) through the Sonderforschungsbereich Transregio 19 "Inflammatory Cardiomyopathy" (SFB TR19) (TP B2) and from the University Hospital Giessen and Marburg Foundation Grant "T cell functionality" (UKGM10/2009). Michel Noutsias has been consultant to the IKDT (Institute for Cardiac Diagnosis and Therapy $\mathrm{GmbH}$, Berlin) in 2004 - 2008, and has received honoraria for presentations and/or participated in advisory boards from Abiomed, AstraZeneca, Bayer, Boehringer Ingelheim, Bristol-Myers Squibb, Fresenius, Miltenyi Biotech, Novartis, Pfizer, and Zoll. The other authors do not have any conflict of interest to declare.

\section{Informed Consent}

The informed consent of the patients involved in the retrospective research was gathered.

\section{Author Contributions}

RDV, MDM, MN and AGR contributed to conceptualization; RDV and CA contributed to formal analysis; RDV and GDB conducted the investigation; GDB contributed to acquiring resources; CA contributed to data curation; RDV, MDM, MN, AGR, CA and GDB contributed to writing and original draft preparation; RDV, MDM, MN, AGR, CA and GDB contributed to writing, review and editing; and RDV contributed to visualization.

\section{References}

1. Roden DM. Mechanisms and management of proarrhythmia. Am J Cardiol. 1998;82(4A):49I-57I.

2. D'Este D, Sorbo MD. [When and how to treat ventricular ectopic beats]. G Ital Cardiol (Rome). 2010;11(10 Suppl 1):21S-26S.

3. Naccarelli GV, Wolbrette DL, Patel HM, Luck JC. Amiodarone: clinical trials. Curr Opin Cardiol. 2000;15(1):6472.

4. Antz M, Bansch D. [Idiopathic dilated cardiomyopathy an appraisal in 2005]. Herz. 2005;30(2):87-90.

5. Arbelo E, Brugada J, Hindricks G, Maggioni AP, Tavazzi L, Vardas P, Laroche C, et al. The atrial fibrillation ablation pilot study: a European Survey on Methodology and results of catheter ablation for atrial fibrillation conducted by the European Heart Rhythm Association. Eur Heart J. 2014;35(22):1466-1478. 
6. Duytschaever M, Demolder A, Phlips T, Sarkozy A, El Haddad M, Taghji P, Knecht S, et al. PulmOnary vein isolation With vs. without continued antiarrhythmic Drug trEatment in subjects with Recurrent Atrial Fibrillation (POWDER AF): results from a multicentre randomized trial. Eur Heart J. 2018;39(16):1429-1437.

7. Pappone C, Rosanio S, Oreto G, Tocchi M, Gugliotta F, Vicedomini G, Salvati A, et al. Circumferential radiofrequency ablation of pulmonary vein ostia: A new anatomic approach for curing atrial fibrillation. Circulation. 2000;102(21):2619-2628.

8. Pokorney SD, Daubert JP. Atrial fibrillation ablation alone or atrial fibrillation ablation plus an antiarrhythmic drug? Eur Heart J. 2018;39(16):1438-1441.

9. Darkner S, Chen X, Hansen J, Pehrson S, Johannessen A, Nielsen JB, Svendsen JH. Recurrence of arrhythmia following short-term oral AMIOdarone after CATheter ablation for atrial fibrillation: a double-blind, randomized, placebo-controlled study (AMIO-CAT trial). Eur Heart J.
2014;35(47):3356-3364.

10. Roux JF, Zado E, Callans DJ, Garcia F, Lin D, Marchlinski FE, Bala R, et al. Antiarrhythmics After Ablation of Atrial Fibrillation (5A Study). Circulation. 2009;120(12):10361040.

11. Kaitani K, Inoue K, Kobori A, Nakazawa Y, Ozawa T, Kurotobi T, Morishima I, et al. Efficacy of antiarrhythmic drugs short-term use after catheter ablation for Atrial Fibrillation (EAST-AF) trial. Eur Heart J. 2016;37(7):610618.

12. Kirchhof P, Benussi S, Kotecha D, Ahlsson A, Atar D, Casadei B, Castella M, et al. 2016 ESC Guidelines for the management of atrial fibrillation developed in collaboration with EACTS. Eur Heart J. 2016;37(38):28932962.

13. Wyse DG, Waldo AL, DiMarco JP, Domanski MJ, Rosenberg Y, Schron EB, Kellen JC, et al. A comparison of rate control and rhythm control in patients with atrial fibrillation. N Engl J Med. 2002;347(23):1825-1833. 